\title{
Sunitinib: 10 years of clinical experience in the treatment of advanced renal cell carcinoma
}

\author{
Anil Kapoor, MD, FRCSC \\ Department of Oncology and Surgery, McMaster University, Associate Editor (Oncology), Canadian Urological Association Journal (CUAJ), Hamilton, ON, Canada
}

Cite as: Can Urol Assoc J 2016;10(11-12Supp17):S235. http://dx.doi.org/10.5489/cuai.4306

$\mathrm{T}$ he arrival of targeted systemic therapies a decade ago transformed the treatment of metastatic renal cell carcinoma (mRCC), which up until that point had been largely resistant to available therapies. For the first time, patients with mRCC had the hope of extended survival.

Since the approval of sunitinib malate by Health Canada in 2006, experience with this particular targeted therapy has grown tremendously. This supplement to the Canadian Urological Association Journal aims to share the accumulated experience with sunitinib in Canada through a series of brief, practical articles reviewing various aspects of the treatment of mRCC.

In the first article, I review the first-line treatment options for mRCC in Canada, which is followed by Dr. Henry Conter's practical overview of the use of sunitinib in the first-line management of RCC in a typical Canadian community practice. Dr. Naveen Basappa then talks about the current treatment options for advanced RCC after firstline treatment with the vascular endothelial growth factor receptor (VEGFR) tyrosine kinase inhibitors. The next three articles provide readers with key insights into dosing and adverse event management with sunitinib. Dr. Christian Kollmannsberger reviews the evidence associating certain side effects of sunitinib as surrogate biomarkers of efficacy; Dr. Sandeep Sehdev provides a practical approach to the management of common toxicities with this agent; and Dr. Georg Bjarnason describes how individualized dosing and scheduling with sunitinib might be the way of the future for optimally managing mRCC. Finally, Dr. Daniel Heng looks forward a decade and provides a glimpse into the future of targeted therapies for mRCC, as new systemic treatments and combination therapies begin to emerge.

We are excited to share with you our first-hand experience with sunitinib and other targeted agents in the management of $m R C C$ and hope you will refer to this supplement often as a practical reference when managing your own patients with mRCC.

Acknowledgement: The authors would like to thank Science and Medicine for editorial assistance with this report.

Correspondence: Dr. Anil Kapoor, McMaster University, Hamilton, ON, Canada; akapoor@mcmaster.ca 